\title{
Cognitive Irrigation System Using Internet of Things based on Wire Less Sensor Network
}

\author{
Madhu Nakirekanti ${ }^{1}$, Dr.R.Murali Prasad ${ }^{2}$, K.Narsimha Reddy ${ }^{3}$ \\ ${ }^{1}$ Assistant Professor, Dept. of ECE, Vardhaman College of Engineering, Hyderabad, Telangana, India. \\ ${ }^{2}$ Professor, Dept. of ECE, Vardhaman College of Engineering, Hyderabad, Telangana, India. \\ ${ }^{3}$ Assistant Professor, Dept. of ECE, Vardhaman College of Engineering, Hyderabad, Telangana, India.
}

\begin{abstract}
In this paper raspberry Pi and Arduino used. Raspberry Pi allows collecting the sensor information from sensor node continuously, store it in a database and providing the web interface to the user. This paper uses Humidity, temperature sensor, soil moisture sensors to detect the water quantity present in agriculture. Arduino using for collecting parameters like Humidity, Soil Moisture Sensor, and Temperature. Aim of this paper is to monitor status of sensors on remote pc through a webpage. Here Humidity, soil moisture sensors, temperature sensors monitored on webpage through Raspberry Pi can ON/OFF the motor using webpage. The Raspberry Pi is connected to the internet. By using the IP address on the web browser, the owner gets a webpage on screen. This page contains all the knowledge concerning the standing of the monitor and might additionally ON/OFF the motor from webpage.
\end{abstract}

Keywords: Raspberry Pi, Arduino, DC motor, Humidity, Soil Moisture Sensors, Temperature Sensors.

\section{Introduction}

Agriculture is that the worldwide prime occupation of soul, sixty fourth of total on the market land is occupied by the agriculture, and it consumes 85 make the most available fresh water. This figure of water consumption increases each year because of globalization and growth. There's a challenge ahead of each country to sustain the fresh foods demand and reducing the farm water consumption.

Irrigation is that the process of watering the soil. The need of water to the soil depends on soil properties like soil moisture and soil temperature. It also depends upon the crop that grows in the soil. From last decade, few existing system operating for reducing the agriculture water consumption, however these systems have some limitations. These systems, watering is completed without analyzing the soil properties, due to that systems apply non uniform water to the soil leads to less yields. Conjointly systems needed a lot of human intervention and time consuming. Therefore we tend to need trendy technology to resolve this drawback and support better irrigation management. For that we've projected system that is web based automatic irrigation system using WSN and embedded Linux board (Raspberry Pi).

The wireless device network creates the networks of multiple devices having capable of computation, communication and sensing. It provides an association between the important world and virtual worlds and having an outsized vary of potential applications of Agriculture, home automation, science, civil infrastructure and security. Throughout this projected system WSN is consists of two nodes, organizer node and Router/End device node. Every node in the main carries with it memory, processor Associate in Nursined an RF transceiver. Raspberry Pi (Rpi) embedded Linux board used as organizer node and Arduino UNO Atmega328 platform used as end device. The operate of the organizer node inside the system is to initiate the communication with distributed finish device nodes via the ZigBee wireless communication protocol and endlessly collects the soil condition and soil temperature data and store collected data within the data. \{The data the info the data\} is created on the raspberry Pi board which may be a MySQL information. Coordinate node analyzes the received information decides the water needed for the soil. If the analyzed information shows that water is needed, the organizer node sends commands to pump controller build Irrigation on. Rpi encompasses a computer network interface and it runs a undemanding information net serve. Then organizer node permits information assortment over ZigBee, and information look and system management from browser remotely.

\section{Wireless Sensor Networks for Conservation and Monitoring Cultural Assets}

The author M. C. Rodríguez-Sánchez, S. Borromeo, Member, IEEE, and J. A. Hernández-Tamames in the paper "Wireless Sensor Networks for Conservation and Monitoring Cultural Assets "w ireless Sensor Networks have Gained a great deal of attention in recent years, particularly with the development of smart sensors . Smart nodes are low-power devices equipped with one or more sensors, a processor, memory, a power supply, a radio chipset, and some kinds of actuators. In this sense, WSNs are oriented to enable the distributed sensing of a physical phenomenon to perform distributed sensing and actuation tasks. On the other hand, 
according to some evaluations of the sensor market, wireless technologies for sensors are increasing its importance in 2010 up to a 60\%. Besides, over the coming 18-24 months WSNs with protocols such as Wifi, Ethernet IP, TCP/IP and others will emerge in importance. These networks have great potential for many applications in monitoring scenarios such as military target tracking and surveillance, natural disaster relief, biomedical health monitoring, and hazardous environment exploration like seismic sensing.

Recently, technology has become more attractive for industry by paving the way to new applications, which go well beyond traditional sensor applications. These applications can be classified in different categories: monitoring, alerting, healthcare assistance, and actuating, among others. A Wireless Sensor Network (WSN) is feasible for the heritage scenarios protection and for monitoring because its specificity, autonomy, self-configurability, lasting lifetime, and mobility. However, their services, protocols, and architectures have constraints to support new issues for monitoring cultural assets: geographical dispersion, insufficient resources, and not enough staff to protect all the monuments and to provide tourist information broadcasting. We propose a modular system. It consists of three elements: a WSN, a "Central System," and a novel platform named "Local Node Gateway." It allows the coexistence of different wireless technologies in order to provide actuation, processing and communication functionalities. This proposal takes a new approach to solve these heritagemonitoring problems.

The architecture is a concept proof in a real scenario oriented to WSN services based on our previous work. It consists of three elements: a WSN, a Central System and a Local Node Gateway. The key issue of this work was the "Local Node Gateway." "Local Node Gateway" is based on modular design. It is designed to provide actuation, processing and communications functionalities. It was developed to help to manage heritage sites using long-range wireless communication, WSN and short-range communications.

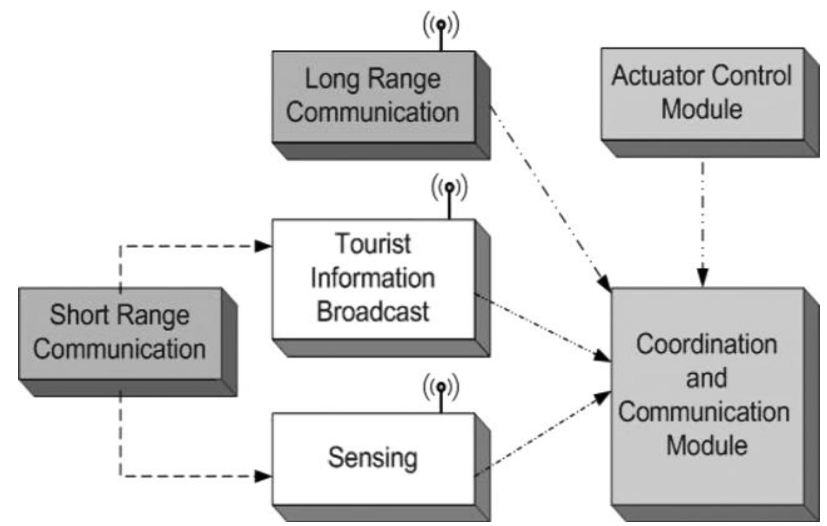

Fig. 2.1 Block diagram for "Local Node Gateway.

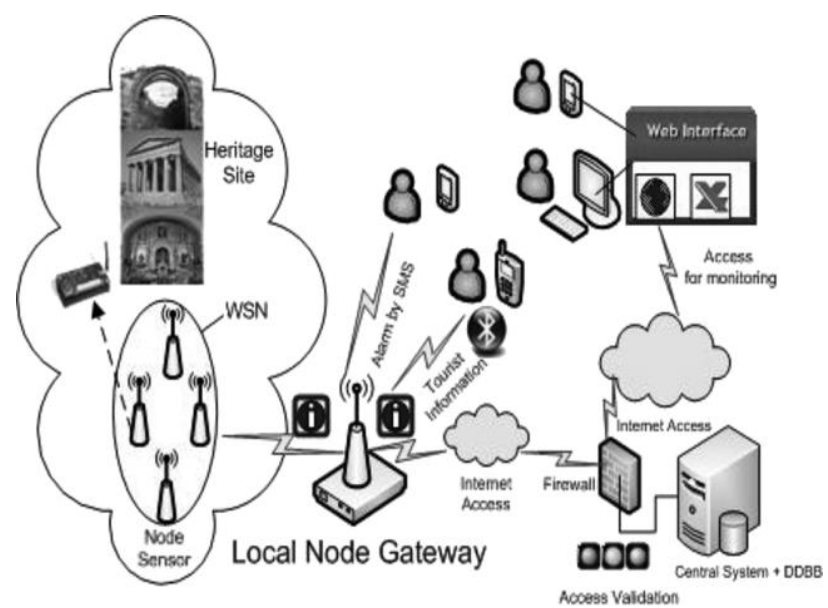

Fig. 2.2 Platform for conservation and monitoring of heritage sites.

This is a gateway between WSN and administrators, who are not on site. These administrators can receive the information from a browser or by Short Message Service (SMS). Furthermore, Local Node Gateway can process data from the environment locally. In this way, we can control lighting, air conditioning and warning systems based on environment data acquisition. Finally, in order to carry out preventive maintenance and remote monitoring we have implemented remote analysis consulting at Central System. 
In this paper, we present a modular system consists of three elements: a WSN, a "Central System," and a novel platform named Local Node Gateway. It allows the existence of different wireless technologies in order to provide actuating, processing, and communication functionalities. Our proposal takes a new approach to solve the heritage monitoring issues: geographical dispersion, insufficient resources, and staff to monitor all the monuments and tourist information distribution. The key issue of our proposal is the implementation of the solution about these problems. All the required functionalities are in the "Local Node Gateway" with a single M2M GSM/GPRS device. It has allowed having a processing and communication unit, without external microprocessor and external embedded devices. It is designed in a modular, flexible, scalable and adaptable way in order to provide sensing, actuating, long range communication and broadcasting information to users. Our proposal allows the coexistence of different protocols based on scenario. With respect to the advantages related to the power consumption, hardware platform size and cost, these are three times better than other commercial systems. Finally, the "Central System" was developed in order to manage and monitor cultural assets by remote control without supervision. It allows carrying out preventive conservation. It consists of a web server, my SQL database and a web interface. Additionally it has been developed using multiplatform and optimal technologies. The proposed system is operative at San Sebastian Church, Seville, Spain, since November 2008. The result was satisfactory to specialists, who studied the collected data in order to make a preventive conservation of this environment. There are several future research projects under way. We are currently planning the development of our own major sensor network to further improve the power consumption and the platform costs. Currently we are working on adding locations based on GPS technology to the WSN systems. This allows monitoring the transport of cultural assets. Furthermore, another important issue is the energy-efficient storage data structure. It is still an open area to explore that requires optimizing some types of database queries, in order to improve the performance and the energy efficiency.

\subsection{Industrial Wireless device Networks: Challenges, style Principles, and Technical Approaches:}

The author "Vehbi C. Gungor, Member, IEEE, and Gerhard P. Hancke, Senior Member, IEEE" within the paper "Industrial Wireless sensing element Networks: challenges, style Principles, and Technical Approaches"stated that the in today's competitive business marketplace, the businesses face growing demands to enhance method efficiencies, befits environmental rules, and meet company money objectives. Given the increasing age of the many industrial systems and therefore the dynamic industrial producing market, intelligent and affordable industrial automation systems area unit needed to enhance the productivity and potency of such systems. Historically, industrial automation systems area unit realized through wired communications. However, the wired automation systems need overpriced communication cables to be put in and often maintained, and thus, they're not wide enforced in industrial plants as a result of their high price. Therefore, there's associate degree imperative would like for efficient wireless automation systems that modify important savings and cut back air-pollutant emissions by optimizing the management of business systems.

The cooperative nature of IWSNs brings many blessings over ancient wired industrial watching and management systems, as well as organization, fast preparation, flexibility, and inherent intelligent-processing capability. During this regard, WSN plays a significant role in making a extremely reliable and self-healing industrial system that speedily responds to time period events with acceptable actions. However, to understand the pictured industrial applications and, hence, take the benefits of the potential gains of WSN, effective communication protocols, which might address the distinctive challenges posed by such systems, area unit needed. With the recent advances in wireless sensing element networks (WSNs), the conclusion of affordable embedded industrial automation systems became possible. In these systems, wireless small sensing element nodes area unit put in on industrial instrumentation and monitor the parameters essential to every equipment's potency supported a mix of measurements like vibration, temperature, pressure, and power quality. These knowledge area unit then wirelessly transmitted to a sink node that analyzes the information from every sensing element. Any potential issues area unit notified to the plant personnel as a complicated warning system. This permits plant personnel to repair or replace instrumentation, before their potency drops or they fail entirely. During this means, harmful instrumentation failures and therefore the associated repair and replacement prices is prevented, whereas yielding with strict environmental rules.

The major technical challenges for realization of IWSNs is printed as follows.

1) Resource constraints: the look and implementation of IWSNs area unit forced by 3 kinds of resources: a) energy b) memory and c) process. Forced by the restricted physical size, sensing element nodes have restricted battery energy provide at a similar time, their recollections area unit restricted and have restricted procedure capabilities.

2) Dynamic topologies and harsh environmental conditions: In industrial environments, the topology and property of the network might vary because of link and sensor-node failures. Moreover, sensors is subject to RF interference, extraordinarily caustic or corrosive environments, high condition levels, vibration, dirt and dust, or 
totally different conditions that challenge performance. These harsh environmental conditions and dynamic network topologies might cause a number of business detector nodes to malfunction.

3) Quality-of-service (QoS) needs: The wide array of applications envisaged on IWSNs will have entirely totally different QoS needs and specifications. The QoS provided by IWSNs refers to the accuracy between the knowledge reportable to the sink node (the management center) and what's really occurring at intervals the commercial sinking, to boot, since detector data unit typically time-sensitive,e.g,alarm notification for the economic facilities, it is vital to receive the knowledge at the sink throughout a timely manner, data with long latency because of method or communication cloud even be superannuated and cause wrong choice with within the looking system.

4) Data redundancy: because of the high density at intervals the configuration, detector observations square measure extraordinarily connected at intervals the world domain to boot, the character of the phenomenon constitutes the temporal correlation between each consecutive observation of the detector node.

5) Packet errors and variable-link capacity: Compared to wired networks, in IWSNs, the possible capability of every wireless link depends on the interference level perceived at the receiver, and high bit error rates (BER $=10-2-10-6)$ area unit determined in communication. Additionally, wireless links exhibit wide variable characteristics over time and house as a result of obstructions and shouting atmosphere

6) Security: Security have to be compelled to be a necessary feature at intervals the fashion of IWSNS to from the communication safe from external denial-of-service (Dos) attacks and intrusion. IWSNs have special characteristics that modification new ways in which of security attacks. Passive attacks unit applied by eavesdropping on transmissions yet as traffic analysis or revelation of message contents. Active attacks embrace modification, fabrication, and interruption that in IWSN cases might embrace node capturing, routing attacks, or flooding.

7) Large-scale activity associate degreed unintended architecture: IWSNs contain an oversized vary of detector nodes (hundreds to thousands or maybe more), that may be unfold haphazardly over the activity field. Moreover, the shortage of planned network infrastructure necessitates the IWSNs to see connections and maintain network property autonomously.

8) Integration with web and totally different networks: it`s of basic importance for the business development of IWSNs to supply service that modify the querying of the network to retrieve useful knowledge from anywhere and at any time. For this reason, the IWSNs have to be compelled to be remotely accessible from internet and, hence, got to be compelled to be integrated with internet Protocol (IP) style. The current sensornetwork platforms use gateways for integration between IWSNs and additionally internet. Note that the' today's detector networks use gateways for integration between IWSNs and additionally internet, the detector nodes might have informatics property at intervals the longer term.

The IWSNs have the potential to enhance productivity of business by providing larger awareness, control, and integration of business process. Despite of the good progress on development of IWSNs, quite few problems still have to be completed to be explored within the future. As an example, associate degree economical readying of IWSNs within the planet is extremely addicted to the flexibility to plan analytical models to gauge and predict. IWSNs performance characteristics, like communication latency and dependability and energy potency. However, attributable to the various industrial-application needs and enormous scale of the network, many technical issues stay to be resolved in analytical IWSN models. Different open problems embrace best sensor-node readying, localization, security, and ability between totally different IWSN makers. Finally, to address RF interference and dynamic/varying wireless-channel conditions in industrial environments, porting a psychological feature radio paradigm to a low-power industrial detector node and developing dominant mechanisms for channel football play is another difficult space nonetheless to be explored.

\subsection{Application of Wireless Sensor Networks for Greenhouse Parameter Control in Precision Agriculture:}

The author "D.D.Chaudhary1, S.P.Nayse2, L.M.Waghmare Sinhgad Institute of Technology, Lonavala, Dist. Pune. MS. India Ph.D. Scholar, Sant Gadgebaba University of Amravati. MS. Republic of India within the paper "Application of Wireless sensing element Networks for Greenhouse Parameter management in preciseness Agriculture" expressed that the within the preciseness Agriculture (PA) varied techniques square measure obtainable to watch and management the desired environmental parameters for the actual crop. It's notably crucial to analyse the strategies which might effectively manage the correct atmosphere. The utilization of wireless sensing element network for the massive space is currently changing into widespread in inexperienced house technology of preciseness agriculture. The parameters of inexperienced house to be management square measure increasing day by day in order that it's going to cause the info traffic and congestion within the future. So that, the wireless sensors derived from PSoC technology with high-bandwidth spectrum or psychological feature radio technology could also be the correct answer for sleek knowledge traffic and remote of inexperienced house from long distance. With the utilization of inexperienced house construct, 
the farmer will manufacture totally different crops in numerous climates and varied seasons. In planned style of the inexperienced house, the farmer will simply keep the specified Crop's atmosphere conditions.

Wireless sensing element nodes square measure terribly little devices that with restricted battery supply. Its process power and memory each are restricted. In automation and management applications, WSN square measure widespread as a result of their ascendible and simple to handle. Now-a-days there square measure variety of economical sensing element nodes square measure obtainable with a high-level technology. They square measure they're capable to gather the environmental knowledge with precise sensors and are able to transmit it to manage station with high potency

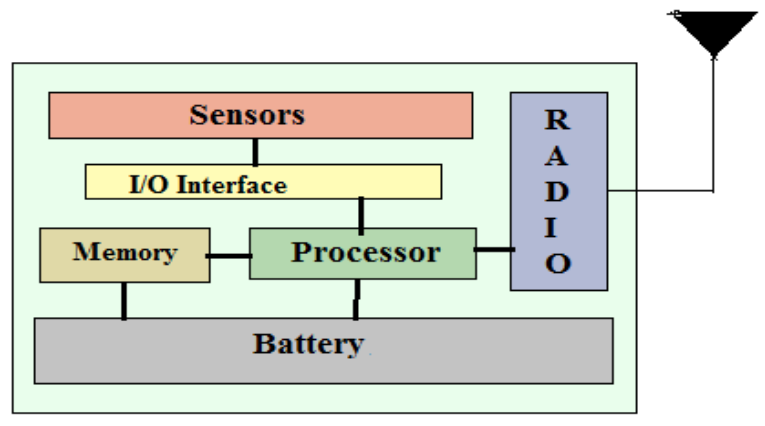

Fig. 2.3 Figure shows Typical Sensor node

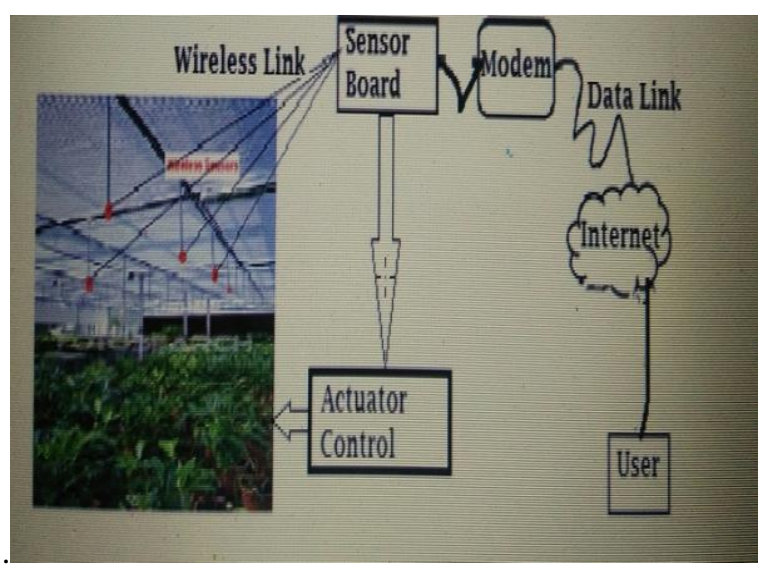

Fig. 2.4 Typical Greenhouse and remote control

In typical inexperienced house management, it's necessary to observe outside wind direction and wind flow. These 2 parameter helps the system to determine the management of fans that are place at totally different places to throw the within air to outside or vice-versa, specified it'll synchronize with outside wind flow. Soil sensors are placed in heavy density as compare to others. Sensors based on time-domain reflectometer (TDR) principal will be used to measure the soil parameter. It is expected to get the soil moisture, soil temp, soil $\mathrm{pH}$ value, and soil electric conductivity. In a real-time environment, we require only two of them, soil temp and soil moisture. This will help to decide either sprinkler or drift irrigation is to be control. Other parameters will help to improve the soil condition through fertilizer or other treatment of the soil. Some of this process is worked in the background of the system. It may just display the parameters and log it for further process or application to decide the soil treatment.

In inexperienced house technology, additional range of the parameters is to be management as a result of, the types of the crop area unit massive. They're increasing day by day thanks to the event in agriculture technology. During this scenario, the wireless detector network with further hardware associate degreed package isan economical answer for inexperienced house management.

Through an experiment it's proved that the hardware develop by cypress opposition is that the best answer that works on low power with less complexness and high dependableness for greenhouse management within the future, if parameter still increase, then for WSN technology with presently obtainable information measure, might not be comfortable. Then WSN with psychological feature radio technology is also the answer. This advancement in exactitude agriculture through wireless detector network in inexperienced house management is extraordinarily helpful. This has scope in developing countries in globe, wherever agriculture is that the main business. 


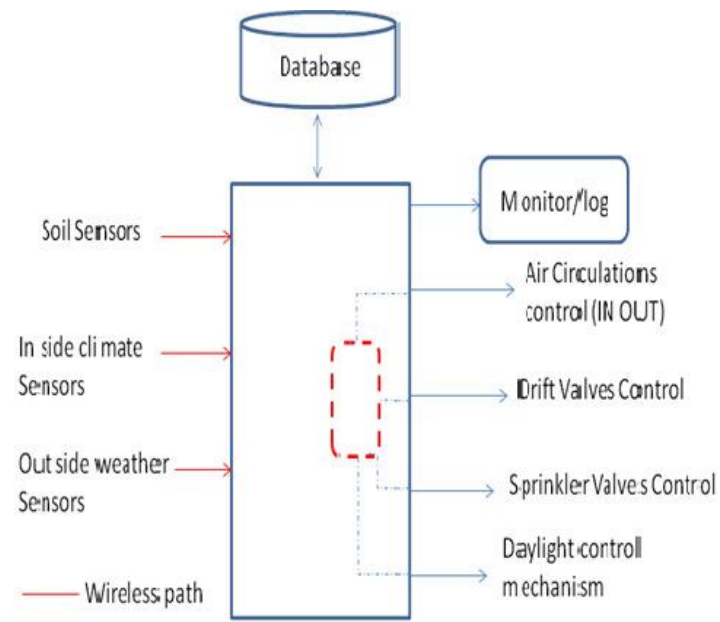

Fig. 2.5 WSN Control blocks Diagram for Green House

The greenhouse climate control is an event-based control system with level crossing sampling technique. In this system, controls are executed in an asynchronous way. The sampling period is governed by system events. This method of sampling consists of transmitting information only when a significant change in the signal occurs which can justify the acquisition of a new sample.

\subsection{Military Applications using Wireless Sensor Networks:}

The author "Ishfaq Ahmad1, Khalil Shah2, Saif Ullah3 Department of computer Science Abdul Wali khan University Mardan1 Khyber Pakhtoonkhwa, Pakistan2" in the paper "Military Applications using Wireless Sensor Networks" that the The rise of Wireless Sensor Networks (WSNs) has brought revolution in the field of technology. These networks comprise of a large number of densely deployed sensor nodes which works through collaboration. In WSNs, each sensor node has limited resources such as, low energy, less bandwidth, limited memory and small computational power. These nodes are very inexpensive in terms of cost, so resource limitation is not a big problem. If a node runs out of energy, so instead of replacing the battery, we can replace the entire node with a new node.

There are different types of sensors available like temperature sensor, humidity sensor, multimedia sensor and others. Due to these variant sensors, WSNs got applications in different fields such as environment monitoring, agriculture monitoring, industrial monitoring, health monitoring, home applications and military operations. Wireless sensing element Networks (WSNs) received monumental attention in recent years thanks to its extraordinary ability of implementation in varied fields. WSNs contains an oversized variety of little sensing element nodes. These nodes are very cheap in terms of cost. In military operations, there is always a threat of being attacked by enemies. So, the use of these cheap sensor nodes will help to reduce the loss. In this paper, we analyze the existing literature of using WSNs for military applications. We will discuss the available scenarios of using sensor nodes in the military uses. We aim to present a better deployment of sensor nodes for military purposes with the help of existing research work. We will try to identify different areas in which we can reduce the damage in case of militant's attack or enemy's outbreak using an intelligent deployment of nodes.

It is clear that WSNs plays an important role in military operations. With the help of these networks, not only the critical areas can be monitored but also due to its flexible nature, it can be expanded to the nearby areas according to the requirements with the passage of time. Moreover due to its fault tolerance characteristic, if any node got damaged, the rest of the network will continue sensing. Rest of the network will not be affected due to the damage of a single or a group of sensors. The use of Wireless Sensor Networks (WSNs) will reduce the casualty rate. Normally these networks are deployed in risky and critical areas where there is always a strong threat to soldiers' in case of their presence. The damage of sensor nodes in that scenario is not noticeable because of their easily availability and inexpensive nature. As we discussed earlier, Wireless Sensor Networks (WSNs) have a vast variety of applications for military purposes, but keeping in mind the importance and critical nature of security and safety, there should be a number of more applications are possible in military operations. This requires further research in this field.

\subsection{Existed method:}

\section{Design and Implementation}

In the existing systems the power generation is carried out by the conventional energy sources. This conventional energy sources causes greenhouse gases emission which leads to the global warming. In the existing system the details about the inventory system like material details, customer details .Consumes more 
time and effort for updating. Reports are not in attractive manner. Efficiency and accuracy cannot be expected. Quick and timely services are not provided slow processing speed. Readymade data retrieval is difficult.

$>$ Semi automatic

$>$ Programming constraints are more

$>$ Data control unit, Data collection unit are available

$>$ High cost

$>$ Maintenance is more

$>$ Fault detection is not easy

\subsection{Proposed system}

In the projected systems the ability generation is meted out by the renewable energy sources.Useful in remote locations wherever a gradual fuel offer isn't doable. Providing an adequate and quality power to domestic and different shoppers

$>$ Full automatic

$>$ Programming constraints are minimum

$>$ Data control Unit, Data collection unit work simply done by remote station server

$>$ Low cost

$>$ Low maintenance

$>$ Fault detection is easy as compared to existing system

For implementing this project we are using the following Hardware Components.

$>$ Raspberry Pi

$>$ Arduino ATmega328p

$>$ NRF module

$>$ Soil Moisture Sensor

$>\mathrm{DTH}$

\section{NODE 1}

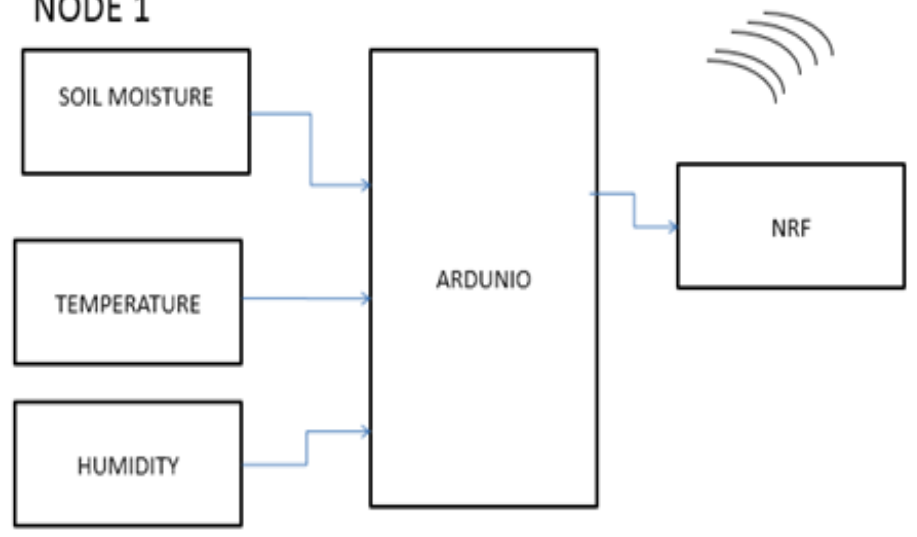

NODE 2

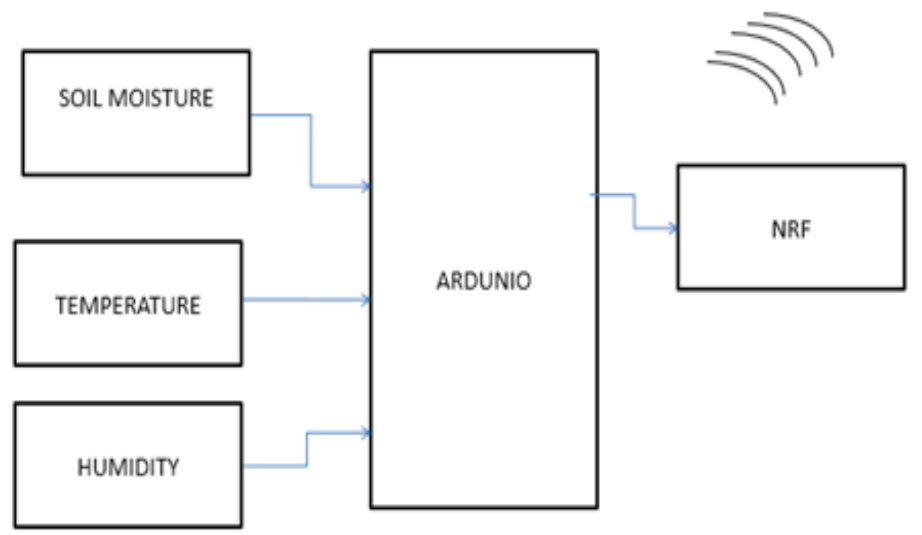

Fig. 3.1 Block diagram of Transmitter section 


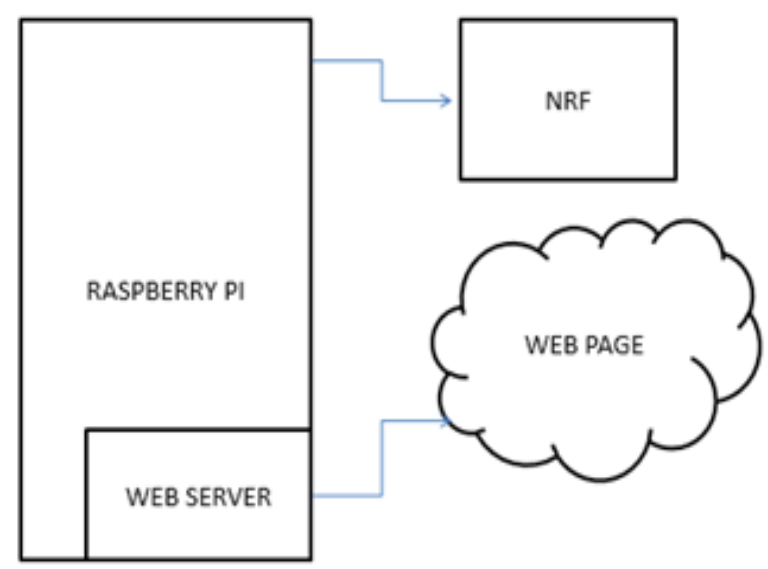

Fig. 3.2 Block diagram of Receiver section

\subsubsection{Raspberry pi}

Raspberry Pi might even be a credit-card sized PC factory-made and designed among the uk by the Raspberry Pi foundation with the intention of teaching basic technology to high school students and each wholly totally different person fascinated by hardware, programming and DIY-Do-it Yourself comes.

The Raspberry Pi is store-bought in three board configurations through licensed manufacturing deals with metropolis element14 (Premier Farnell), RS components and Egoman. These companies sell the Raspberry $\mathrm{Pi}$ on-line. Egoman produces a version for distribution entirely in China and Taiwan that will be distinguished from utterly wholly totally different Pies by their red coloring and lack of FCC/CE marks. The hardware is that the same across all makers.

The Raspberry Pi encompasses a Broadcom BCM2835 system on a chip (SoC), that includes Associate in Nursing ARM1176JZF-S 700 rate processor, Video Core IV GPU and was originally shipped with 256 megabytes of RAM, later upgraded (Model B \&amp; Model B+) to 512 MB. It doesn't embody a in and of itself disk or solid-state drive, however it uses Associate in Nursing Coyote State card for booting and chronic storage, with the Model B+ employing a small low Coyote State. The muse provides Debian and Arch UNIX system OS package ARM distributions for transfer. Tools unit getable for Python as a results of the foremost language, with support for BBC BASIC (via the reduced instruction set computing OS image or the liquor Basic clone for Linux), C, Java and Perl

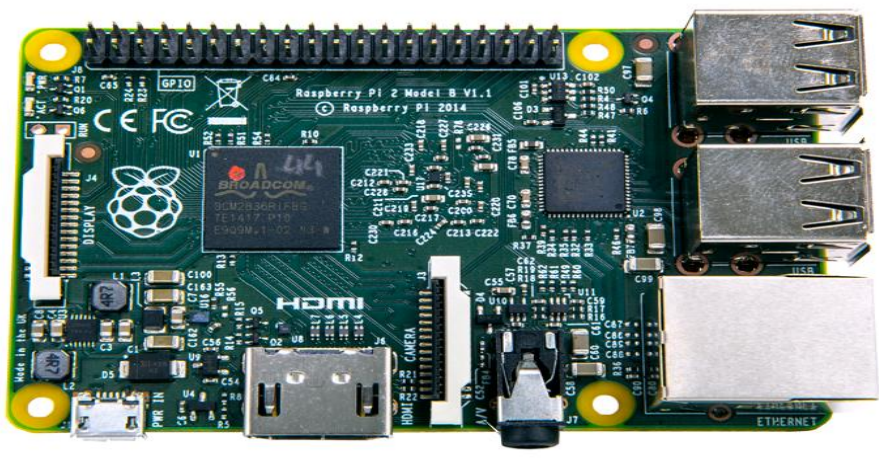

Fig. 3.3 Figure shows Raspberry Pi board

\section{Using the Template}

First the sensors are deployed within the farm. The gap between the 2 sensing element node is depends on the kind of soil. The mix of Arduino, nrf, and wet sensing element is liable for capturing the wet gift in soil. Betting on the wet contain and temperature of soil the Watering to the crop is given. The soil parameters are keep in info tables and these tables are displayed on internet the online the net page victimization PHP script and web server. 


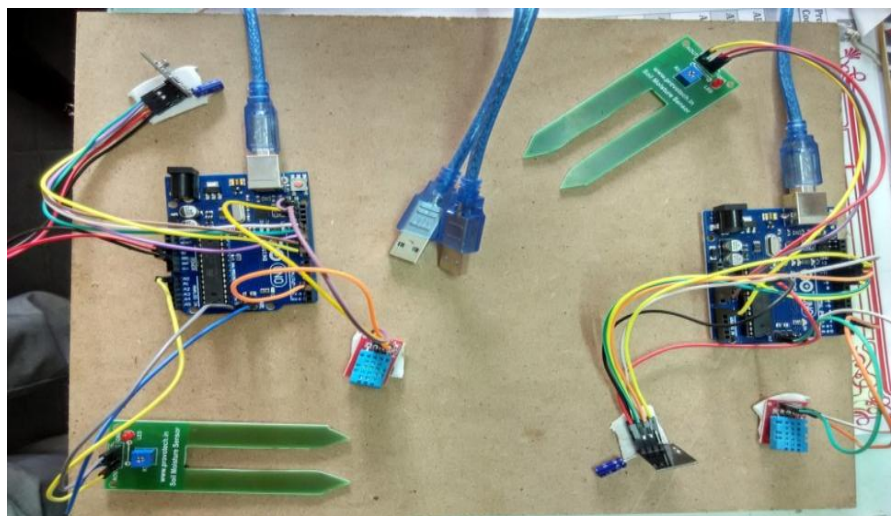

Fig. 4.1 Figure shows Transmitter section hardware

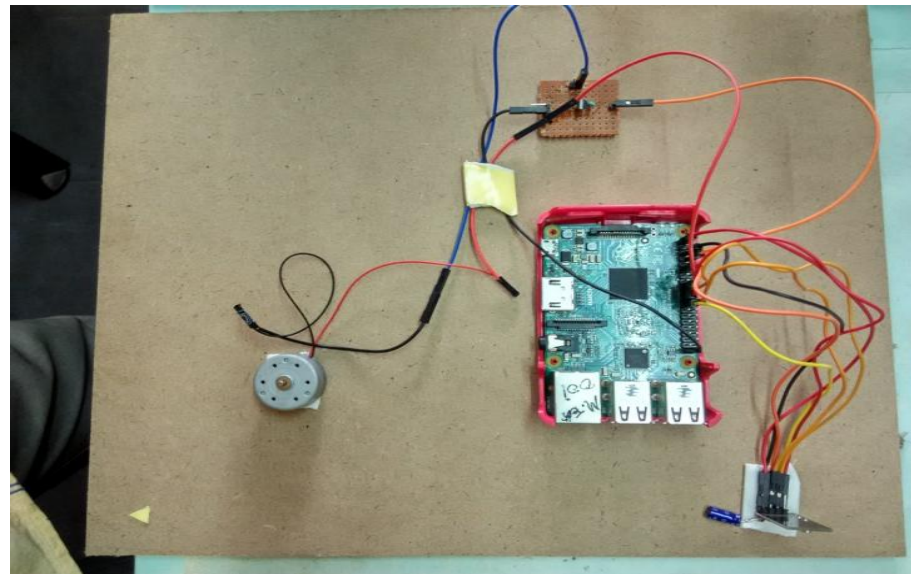

Fig.4.2 Figure shows Receiver section hardware

Then open the webpage by enter in IP address. Then the webpage is displayed as shown Then the webpage is displayed as shown in the following figure the irrigation monitoring system shows that Pump ON and OFF Functions in the following Webpage Fig.4.The SmarAutomation system also shown in the figure.

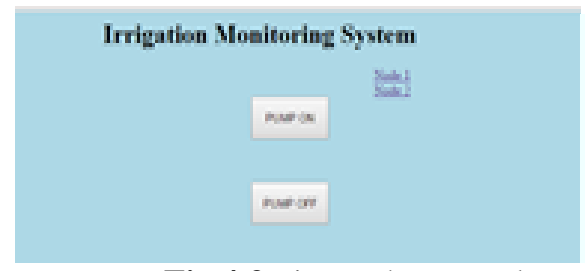

\section{Irrigation Monitoring System}

weleome to smart Avfomation system :

Fig.4.3 Figure shows Webpage is displayed as shown above

The Internet of things Implementation is as shown in the figure.the updated values on node 1 and node4 are shown in the Fig.5

Internet of Things Inplementation

The Fump bu bers Taraed ov

\section{Internet of Things Inplementation}

The Pane hat besa Tereed ory

Fig.4.4 Figure Shows Updated values on node1 and node4

The Updated values on the nodes are shown in the putty window as shown in the Fig.4.5 


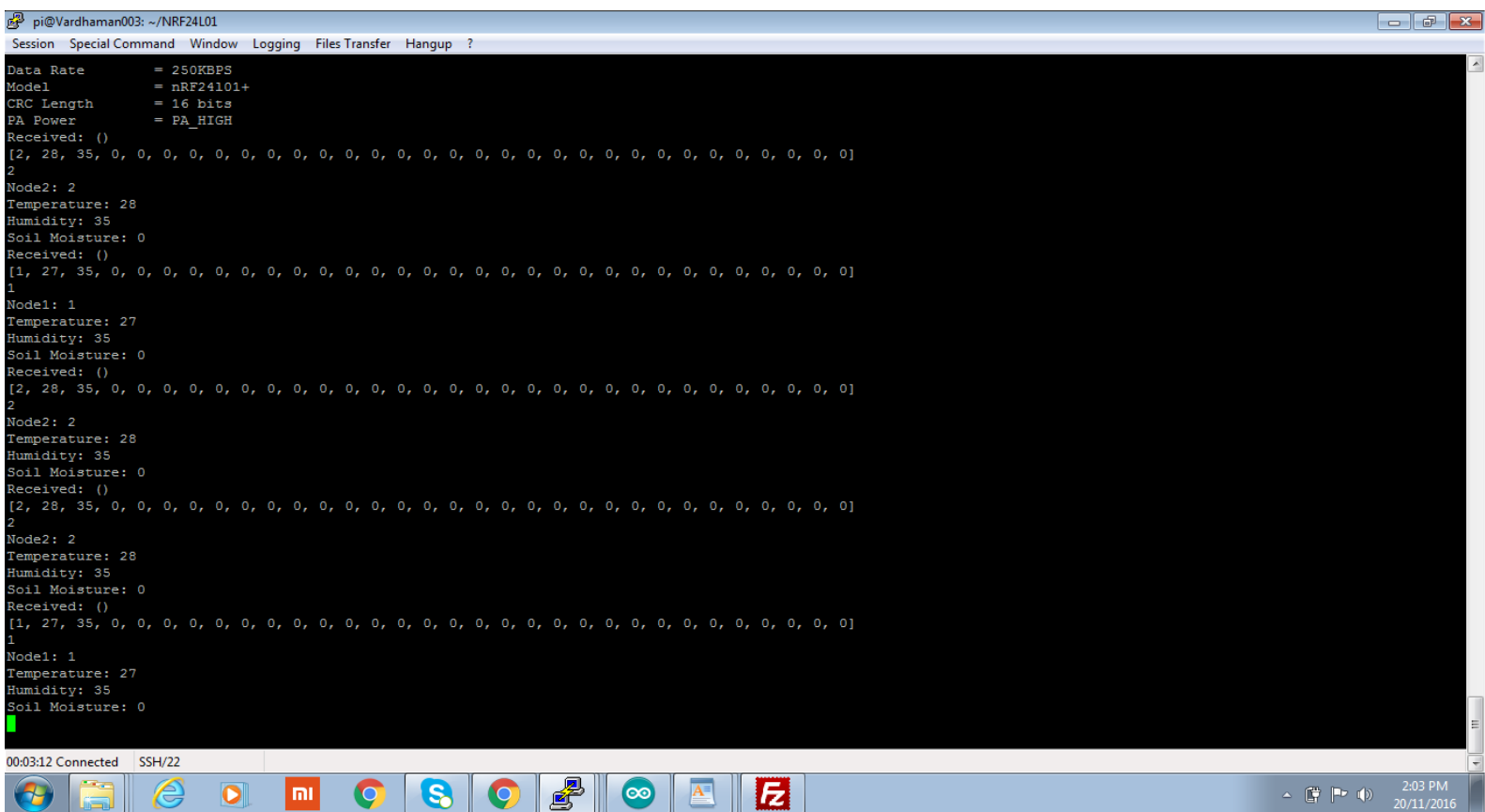

4.8 Figure shows Updated values on putty window

\section{Conclusion}

This paper styles the machine-controlled wireless irrigation system victimization WSN and embedded Linux board. During this we've got used raspberry Pi as associate degree embedded Linux board that permits grouping the detector info from detector node unceasingly, store it in a very info and providing the online interface to the user. The system is watering to the crop uniform by analyzing the soil parameters, it'll facilitate to scale back the $\mathrm{H} 2 \mathrm{O}$ consumption. By providing the online interface and automation user will simply monitor the system and it'll minimize the human intervention. The NRF module is employed here for wireless communication it'll produce network simply and combination of Arduino, NRF module and detector produce a coffee power cheap detector node. The Apache net server crated on Raspberry Pi simply displaying the contents of detector knowledge.

\section{References}

[1]. Gutierrez, J: Villa-Medina, J.F; Nieto-Garibay; Porta-Gandara, M.A, "Automated Irrigation System Using a Wireless Sensor Network and GPRS Module” Instrumentation and Measurement, IEEE Transaction on, vol.63, no.1, pp.166, 176, Jan 2014.

[2]. Yunseop Kim; Evans, R.G.; Iversen, W.M., "Remote Sensing and Control of an Irrigation System Using a Distributed Wireless Sensor Network," Instrumentation and Measurement, IEEE Transactions on, vol.57, no.7, pp.1379, 1387, July 2008.

[3]. Wark, T.; Corke, P.; Sikka, P.; Klingbeil, L.; Ying Guo; Crossman, C.; Valencia, P.; Swain, D.; Bishop-Hurley, G., "Transforming Agriculture through Pervasive Wireless Sensor Networks," Pervasive Computing, IEEE, vol.6, no.2, pp.50,57, April-June 2007.

[4]. Rani, M.U.; Kamalesh, S., "Web based service to monitor automatic irrigation system for the agriculture field using sensors," Advances in Electrical Engineering (ICAEE), 2014 International Conference on , vol., no., pp.1,5, 9-11 Jan. 2014.

[5]. Genghuang Yang; Yuliang Liu; Li Zhao; Shigang Cui; Qingguo Meng; Chen HongDa, "Automatic irrigation system based on wireless network," Control and Automation (ICCA), 2010 8th IEEE International Conference on , vol., no., pp.2120,2125, 9-11 June 2010.

[6]. J. M. Blonquist, Jr., S. B. Jones, and D. A. Robinson, "Precise irrigation scheduling for turfgrass using a subsurface electromagnetic soil moisture sensor," Agricult. Water Manag. vol. 84, nos. 1-2, pp. 153-165, Jul. 2006.

[7]. O. M. Grant, M. J. Davies, H. Long bottom, and C. J. Atkinson, "Irrigation scheduling and irrigation systems: Optimizing irrigation efficiency for container ornamental shrubs," Irrigation Sci., vol. 27, no. 2, pp. 139-153, Jan. 2009.

[8]. Y. Kim, R. G. Evans, and W. M. Iversen, "Remote sensing and control of an irrigation system using a distributed wireless sensor network," IEEE Trans. Instrum. Meas., vol. 57, no. 7, pp. 1379-1387, Jul. 2008.

[9]. Y. Kim and R. G. Evans, "Software design for wireless sensor-based site-specific irrigation," Comput. Electron. Agricult. vol. 66, no. 2, pp. 159-165, May 2009.

[10]. D. K. Fisher and H. A. Kebede, "A low-cost microcontroller-based system to monitor crop temperature and water status," Comput. Electron. Agricult, vol. 74, no. 1, pp. 168-173, Oct. 2010.

[11]. V. C. Gungor and G. P. Hancke, "Industrial wireless sensor networks: Challenges, design principles, and technical approaches," IEEE Trans. Ind. Electron., vol. 56, no. 10, pp. 4258-4265, Oct. 2009.

[12]. A. Araujo, J. Garcia-Palacios, J. Blesa, F. Tirado, E. Romero, A. Samartin, and O. Nieto-Taladriz, "Wireless measurement system for structural health monitoring with high time-synchronization accuracy,” IEEE Trans. Instrum. Meas., vol. 61, no. 3, pp. 801-810, Mar. 2012.

[13]. H.-C. Lee, A. Banerjee, Y.-M. Fang, B.-J. Lee, and C.-T. King, "Design of a multifunctional wireless sensor for in-situ monitoring of debris flows," IEEE Trans. Instrum. Meas., vol. 59, no. 11, pp. 2958-2967, Nov. 2010.

[14]. M. R. Frankowiak, R. I. Grosvenor, and P. W. Prickett, "A review of the evolution of microcontroller-based machine and process monitoring,” Int. J. Mach. Tool Manuf., vol. 45, nos. 4-5, pp. 573-582, Apr. 2005 
[15]. R. Yan, H. Sun, and Y. Qian, "Energy-aware sensor node design with its application in wireless sensor networks," IEEE Trans. Instrum. Meas., vol. 62, no. 5, pp. 1183-1191, May 2013.

[16]. E. Sardini and M. Serpelloni, "Self-powered wireless sensor for air temperature and velocity measurements with energy harvesting capability,” IEEE Trans. Instrum. Meas., vol. 60, no. 5, pp. 1838-1844, May 2011

[17]. C. Kompis and P. Sureka, "Power management technologies to enable remote and wireless sensing," ESP KTN, Teddington, U.K., Tech. Rep., May 2010. [44] M. T. Penella and M. Gasulla, "Runtime extension of low-power wireless sensor nodes using hybridstorage units," IEEE Trans. Instrum. Meas., vol. 59, no. 4, pp. 857-865, Apr. 2010.

[18]. N. Wang, N. Zhang, and M. Wang, "Wireless sensors in agriculture and food industry-Recent development and future perspective," Comput. Electron. Agricult, vol. 50, no. 1, pp. 1-14, Jan. 2006.

[19]. P. Corke, T. Wark, R. Jurdak, H. Wen, P. Valencia, and D. Moore, "Environmental wireless sensor networks," Proc. IEEE, vol. 98, no. 11, pp. 1903-1917, Nov. 2010.

[20]. D. D. Chaudhary, S. P. Nayse, and L. M. Waghmare, "Application of wireless sensor networks for green house parameters control in precision agriculture," Int. J. Wireless Mobile Netw., vol. 3, no. 1, pp. 140-149, Feb. 2011.

[21]. P. Mariño, F. P. Fontan, M. A. Dominguez, and S. Otero, "An experimental ad-hoc WSN for the instrumentation of biological models,” IEEE Trans. Instrum. Meas., vol. 59, no. 11, pp. 2936-2948, Nov. 2010. 